\title{
Preventing transmission of bloodborne viruses from infected healthcare workers to patients: Summary of a new Canadian Guideline
}

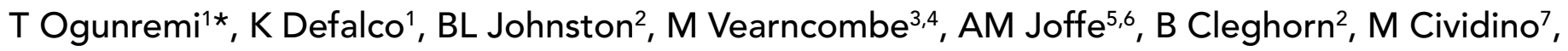 \\ DK Wong ${ }^{3,8}$, T Mazzulli ${ }^{3,9}$, J Wong ${ }^{10,11}$, MA Isinger ${ }^{12}$, Y Robert ${ }^{13}$, I Boucoiran ${ }^{14}$, K Dunn ${ }^{1}$, B Henry ${ }^{11,15}$
}

\begin{abstract}
Background: Although it is well documented that bloodborne viruses (BBVs), including human immunodeficiency virus (HIV), hepatitis $C$ virus ( $\mathrm{HCV}$ ) and hepatitis $B$ virus (HBV) have been transmitted from patients to healthcare workers (HCWs), there has also been reported transmission from HCWs to patients during the provision of health care. With remarkable progress in infection prevention, diagnosis tools, treatment regimens and major improvements in guideline development methodology, there was a need to develop an evidence-based guideline to replace the 1998 Canadian consensus document for managing HCWs infected with BBVs.
\end{abstract}

Purpose: This article summarizes the Canadian Guideline on the Prevention of Transmission of Bloodborne Viruses from Infected Healthcare Workers in Healthcare Settings.

Methods: A Guideline Development Task Group was established and key questions developed to inform the guideline content. Systematic reviews were conducted to evaluate the risk of $\mathrm{HCW}$-to-patient transmission of HIV, HCV and HBV. Environmental scans were used to provide information on Expert Review Panels, disclosure of a HCW's serologic status and lookback investigations. Federal, provincial and territorial partners and key stakeholder organizations were consulted on the Guideline.

Results: The risk of HCW-to-patient BBV transmission was found to be negligible, except during exposure-prone procedures, where there is a risk that injury to the HCW may result in exposure of a patient's open tissues to the HCW's blood. Risk of ensuing transmission and the rate of transmission varied by BBV, and were lowest with HIV and highest with HBV. The Guideline provides key content, including recommendations regarding criteria to determine if a procedure is an exposure-prone procedure, management of HCWs infected with a BBV, including considerations for the HCW's fitness for practice, Expert Review Panels, HCW disclosure obligations and right to privacy and lookback investigations.

Conclusion: This new Guideline provides a pan-Canadian approach for managing HCWs infected with a BBV, with recommendations related to preventing $\mathrm{HCW}$-to-patient transmission of BBVs during the provision of care.
This work is licensed under a Creative Commons Attribution 4.0 International License.

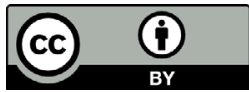

Affiliations

${ }^{1}$ Centre for Communicable Diseases and Infection Control, Public Health Agency of Canada, Ottawa, ON

2 Dalhousie University, Halifax, NS

${ }^{3}$ University of Toronto,

Toronto, ON

${ }^{4}$ Sunnybrook Health Sciences

Centre, Toronto, ON

${ }^{5}$ University of Alberta,

Edmonton, $A B$

${ }^{6}$ Alberta Health Services,

Edmonton, $\mathrm{AB}$

${ }^{7}$ St. Joseph's Healthcare

Hamilton, Hamilton, ON

${ }^{8}$ Toronto General Hospital, Toronto, ON

${ }^{9}$ Mount Sinai Hospital,

Toronto, ON

${ }^{10}$ BC Centre for Disease Control, Vancouver, BC

${ }^{11}$ University of British Columbia, Vancouver, BC

${ }^{12}$ Clinical Ethicist, Ottawa, ON

${ }^{13}$ Collège des médecins du Québec, Montréal, QC

${ }^{14}$ Centre hospitalier universitaire Sainte-Justine, Montréal, QC

${ }^{15}$ British Columbia Ministry of Health, Victoria, BC

\section{*Correspondence:}

toju.ogunremi@canada.ca

Suggested citation: Ogunremi T, Defalco K, Johnston BL, Vearncombe M, Joffe AM, Cleghorn B, Cividino M, Wong DK, Mazzulli T, Wong J, Isinger MA, Robert Y, Boucoiran I, Dunn K, Henry B. Preventing transmission of bloodborne viruses from infected healthcare workers to patients: Summary of a new Canadian Guideline. Can Commun Dis Rep 2019;45(12):317-22. https://doi.org/10.14745/ccdr.v45i12a03

Keywords: bloodborne viruses, HIV, hepatitis C, hepatitis B, transmission, bloodborne pathogens, healthcare workers, exposure-prone procedure, guideline, recommendations, Canada 


\section{Introduction}

The purpose of the new Guideline on the Prevention of Transmission of Bloodborne Viruses from Infected Healthcare Workers in Healthcare Settings (hereafter called Guideline) (1) is to provide a national framework for developing policies and procedures to prevent the transmission of bloodborne viruses (BBVs), specifically human immunodeficiency virus (HIV), hepatitis $C$ virus (HCV) and hepatitis $B$ virus (HBV), from infected healthcare workers (HCWs) to patients in the Canadian healthcare setting. The Guideline was developed by the Public Health Agency of Canada (PHAC) with technical expertise provided by a Guideline Development Task Group (Task Group) of the National Advisory Committee on Infection Prevention and Control (NAC-IPC) (2). This Guideline replaces Health Canada's 1998 Proceedings of the Consensus Conference on Infected Health Care Workers: Risk for Transmission of Bloodborne Pathogens (3). This article summarizes the development, key content and recommendations of the Guideline.

The Guideline assumes that HCWs will adhere to Routine Practices when providing care to all patients at all times and in all settings (4). Failure to adhere to infection prevention and control principles identified as Routine Practices could result in transmission of BBVs. For HCWs who perform exposure-prone procedures, there is a risk of percutaneous injury and therefore a subsequent risk of patient exposure to the HCW's blood. Our review of the worldwide literature identified several reports of $\mathrm{HCW}$-to-patient transmission of BBVs in healthcare settings (5-7). Transmission incidents from the 1980s and 1990s highlighted the need for policies and guidelines internationally with a goal to minimize the risk of transmission. In defining the risk of transmission of a BBV from an infected HCW to a patient, both the actual risk determined from available evidence, and the risk perceived by the public inform what is considered to be acceptable risk. While zero risk of transmission is unattainable, the availability of a vaccine that prevents HBV infection, effective treatment for HCV resulting in a sustained virologic response and suppression of HIV with strict adherence to antiviral therapy could render transmission risks from these BBVs negligible.

The Guideline provides a comprehensive summary of relevant background information, current evidence and recommendations to inform the prevention of transmission of BBVs from $\mathrm{HCWs}$ to patients while providing care.

\section{Methods}

\section{Stakeholder consultation and scope}

A preliminary consultation with key partner and stakeholder organizations was conducted prior to the development of the Guideline. This involved collating feedback via a needs assessment to inform the Guideline scope and key issues.
A project protocol was developed to outline the steps and methods for conducting systematic reviews and environmental scans necessary to address issues within the scope of the guideline and inform the recommendations provided. Key questions were developed to address issues identified. These questions were informed by conducting six systematic reviews, one narrative review, and three environmental scans.

Consultation with relevant organizations was ongoing as needed during the development of the Guideline, and a final broad consultation with all pertinent partners, key stakeholder organizations and subject matter experts was conducted upon completion of a full draft of the Guideline.

\section{Review of epidemiologic investigations}

Worldwide reports of potential BBV exposure via an infected $\mathrm{HCW}$ (with or without transmission to patients) were reviewed to help identify factors that influence the risk of percutaneous injury to $\mathrm{HCW}$ s and the risk of BBV transmission to patients, given HCW injury.

The approach to determining risk associated with procedures reported in the epidemiologic investigations and the categorization of exposure-prone procedures in key international guidelines were reviewed to inform the definition for exposureprone procedures in the Guideline.

\section{Systematic reviews}

Six systematic reviews (encompassing literature from 1995 to 2016) were conducted for key questions to specifically evaluate factors affecting the risk of transmission of HIV, HCV and HBV from infected HCWs to patients and examine infectivity of each virus related to the source serum viral load at time of exposure. Four databases were reviewed: Ovid MEDLINE; EMBASE; Global Health; and Scopus. Pre-identified screening criteria helped determine studies (published in English and French) that were eligible to inform relevant sections in the Guideline. Critical appraisal of eligible studies and grading of evidence was conducted using PHAC's Critical Appraisal Tool Kit (8). The evidence from eligible studies was reviewed and summarized. Complete details of the key questions, study eligibility criteria and findings from the systematic reviews for each BBV will be published in separate articles.

A narrative review of relevant published studies, including a systematic review of randomized controlled trials (9), was conducted to inform a key question regarding the clinical effectiveness of double gloving.

\section{Environmental scans}

Environmental scans were conducted to address key questions where the topic dealt with organizational, regulatory, and/ or ethical issues not solely or directly informed by scientific research. These provided background information and helped identify the general consensus of opinion internationally regarding Expert Review Panels, disclosure of a HCW's 
serologic status, and conducting lookback investigations. The environmental scans involved identifying and reviewing relevant documents obtained from a search of grey literature or articles outside of the indexed medical literature, including regulatory authorities' and professional associations' policy documents, other international and provincial guidelines and reports from government institutions.

\section{Technical expertise for evidence reviews and developing recommendations}

Technical expertise for review of the evidence around risk factors for transmission, including definition of an exposure-prone procedure and development of recommendations, were provided by the Task Group. The group was composed of members with expertise in infectious diseases, medical microbiology and virology, infection prevention and control, public health, occupational health, hepatology, dentistry, medical ethics and obstetrics and gynecology (refer to the Guideline for list of names and affiliations of group members) (1). The Task Group reported to the NAC-IPC.

\section{Findings}

\section{Stakeholder consultation and scope}

Feedback from the preliminary stakeholder consultation (needs assessment) identified the key issues for inclusion in a national guideline on this topic (Figure 1).

Figure 1: Key issues and/or controversies regarding the management of a healthcare worker infected with a bloodborne virus

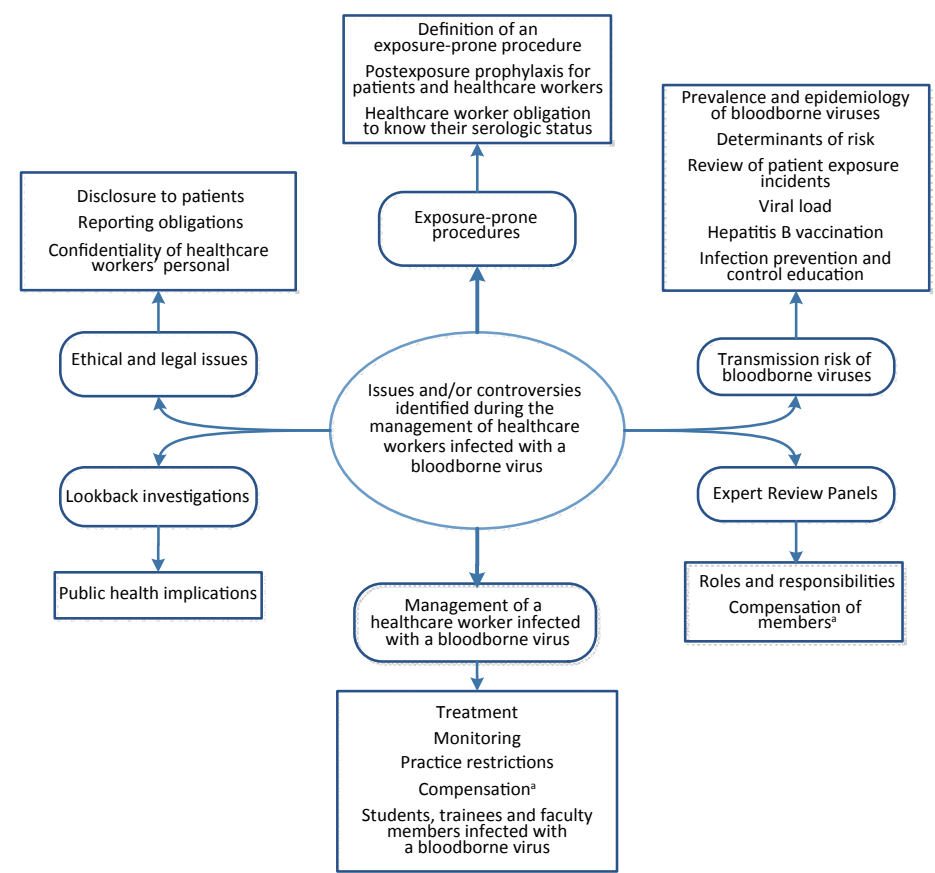

a The issue of compensation of healthcare workers and Expert Review Panel members is beyond the scope of the Guideline
All feedback received from the final broad consultation was reviewed and addressed as appropriate by the Task Group, prior to finalizing the Guideline. The list of all partners, organizations and subject matter experts who were invited to provide feedback is provided in the Guideline (1).

\section{Review of epidemiologic investigations}

Factors affecting the risk of percutaneous injury to HCWs included the type and duration of procedure performed, surgical techniques and expertise and compliance with infection prevention and control practices. Factors affecting risk of transmission included the nature of the injury and exposure, frequency of injury, size of inoculum or volume of blood present in an exposure incident, viral load and clinical status of the source and susceptibility of the exposed individual.

Other factors affecting transmissibility include communicability of the BBV and immunologic status of the HCW. Identified investigations reporting on potential patient exposure to $\mathrm{HCWs}$ infected with HIV, HCV or HBV are summarized in Table 1. The number of exposure incidents involving transmission was lowest for HIV and highest for HBV.

Table 1: Epidemiologic investigations involving potential patient exposure to a bloodborne virus during procedures performed by infected healthcare workers

\begin{tabular}{|l|r|r|r|r|}
$\begin{array}{c}\text { Virus } \\
\text { (publication } \\
\text { year) }\end{array}$ & $\begin{array}{c}\text { Number of } \\
\text { investigations } \\
\text { with no } \\
\text { transmission }\end{array}$ & $\begin{array}{c}\text { Number of } \\
\text { investigations } \\
\text { with } \\
\text { transmission }\end{array}$ & $\begin{array}{c}\text { Number } \\
\text { of } \\
\text { patients } \\
\text { infected }\end{array}$ & \multicolumn{1}{|c|}{$\begin{array}{c}\text { Total } \\
\text { number of } \\
\text { investigations }\end{array}$} \\
\hline $\begin{array}{l}\text { HIV } \\
(1985-2017)\end{array}$ & 45 & 4 & 9 & 49 \\
\hline $\begin{array}{l}\text { HCV } \\
(1996-2013)\end{array}$ & $7 \mathrm{a}$ & 24 & $68^{\mathrm{a}}$ & 31 \\
\hline $\begin{array}{l}\text { HBV } \\
(1986-2013)\end{array}$ & 3 & 29 & 237 & 32 \\
\hline Total & 55 & 57 & 314 & 112 \\
\hline
\end{tabular}

Abbreviations: $\mathrm{HBV}$, hepatitis $\mathrm{B}$ virus; $\mathrm{HCV}$, hepatitis $\mathrm{C}$ virus; $\mathrm{HIV}$, human immunodeficiency virus a Excludes investigations that reported illicit drug use by a healthcare worker infected with hepatitis $C$ virus

\section{Exposure-prone procedures}

Exposure-prone procedures are defined as invasive procedures where there is a risk that injury to the HCW may result in the exposure of the patient's open tissues to the blood of the HCW. Exposure-prone procedures vary from specialty to specialty. For transmission of a BBV from an infected HCW to patient to occur during an exposure-prone procedure, three conditions are necessary:

- The HCW must sustain an injury or have a condition that allows for exposure

- The HCW's blood must come in contact with a patient's wound, traumatized tissue, mucous membranes or similar portal of entry

- $\quad$ The HCW must be sufficiently viremic 


\section{Systematic reviews}

Eligible studies mainly comprised of reported exposure incidents with or without ensuing transmission. These helped identify risk factors for BBV transmission and thus best practice to prevent transmission. Findings from the systematic reviews were analyzed by the Task Group. This analysis as well as expert opinion where gaps existed, informed the development of recommendations. Publication of the full systematic reviews is pending and not included in this summary.

\section{Guideline recommendations: HIV, $\mathrm{HCV}$ and HBV}

The following recommendations are common to HCWs infected with any of the three BBVs (refer to the Guideline for full context and footnotes on these):

- $\quad$ All HCWs who perform exposure-prone procedures have ethical and professional obligations to know their HIV/HCV/ HBV status

- If their status is negative, the HCWs should be tested at appropriate intervals: as determined by their level of risk and whenever an exposure has occurred

- $\mathrm{HCW}$ infected with HIV/HCV/HBV who do not perform exposure-prone procedures do not need any restrictions on practice based on their BBV status alone

- If a HCW-to-patient transmission of HIV/HCV/HBV occurs, the HCW should cease clinical practice immediately until determination for fitness to return to practice is made Recommendations specific to HCWs infected with HIV, HCV or $\mathrm{HBV}$ are summarized in Tables 2, 3 and 4 respectively.

\section{Table 2: Recommendations for management of healthcare workers infected with HIV}

\section{Recommendations}

HCWs infected with HIV should seek medical care from a physician with expertise in HIV management for optimal health maintenance and should be managed according to current recommendations with regular monitoring of HIV RNA levels.

HCWs infected with HIV should be restricted from performing exposure-prone procedures until:

- the HCW is under the care of a physician with expertise in HIV management; and

- the HCW is either on effective combination antiretroviral therapy or has been identified as an elite controller; and

- the HCW's viral load is undetectable ${ }^{\text {a }}$.

HCWs infected with HIV who are on effective combination antiretroviral therapy (or are elite controllers), and have an undetectable viral load should have no restrictions on practice based on HIV status alone.

Abbreviations: HCW, healthcare worker; HIV, human immunodeficiency virus; RNA, ribonucleic acid

a There are variations in minimum detectable viral load thresholds for different assays. In addition, there may be situations where very low viral loads and/or transient blips (up to 400 copies $/ \mathrm{mL}$ ) may occur. These blips may not be clinically relevant and thus do not indicate treatment failure and will not necessarily trigger practice restrictions or lookback investigations. However, some jurisdictions may manage transient blips by maintaining stricter or more conservative thresholds
Table 3: Recommendations for management of healthcare workers infected with hepatitis $C$ virus

\section{Recommendations}

Confirmation of active HCV infection should be done using HCV RNA testing. HCWs infected with HCV should seek medical care from a physician with expertise in HCV management for optimal health maintenance and should be managed according to current recommendations.

HCWs testing positive for HCV RNA should be restricted from performing exposure-prone procedures until:

- the HCW is under the care of a physician with expertise in $\mathrm{HCV}$ management; and

- the HCW has completed effective therapy ${ }^{\mathrm{a}, \mathrm{b}}$; and

- the HCW has tested negative for HCV RNA at least 12 weeks post-treatment ${ }^{\mathrm{b}}$.

Note: Expert Review Panels may individualize practice restrictions to allow a HCW to perform exposure-prone procedures while on effective therapy provided the virus is undetectable. The HCW's practice should then be restricted post treatment until a sustained virologic response is confirmed.

HCWs testing negative for HCV RNA 12 weeks post-treatment can be considered to have a sustained virologic response and should have no restrictions on practice based on $\mathrm{HCV}$ status alone.

Abbreviations: $\mathrm{HCV}$, hepatitis $\mathrm{C}$ virus; $\mathrm{HCW}$, healthcare worker; $\mathrm{RNA}$, ribonucleic acid a Due to the availability of effective therapy for HCV with sustained virologic response, this guideline does not recommend a serum HCV RNA cut off level for practice restrictions as recommended in other guidelines

${ }^{b}$ The overarching principle for management of $\mathrm{HCWs}$ infected with $\mathrm{HCV}$ who perform exposure-prone procedures is to restrict practice while the virus is detectable

\section{Table 4: Recommendations for management of healthcare workers infected with hepatitis B virus}

\section{Recommendations}

$\mathrm{HCW}$ s who remain susceptible to $\mathrm{HBV}$ (anti-HBs negative and anti-HBc negative) should be tested at appropriate intervals as determined by their level of risk and whenever an exposure has occurred.

$\mathrm{HCWs}$ born or previously residing in high HBV endemic countries should be tested for both anti-HBc and HBsAg to fully define HBV status $^{\mathrm{a}, \mathrm{b}}$.

HCWs infected with HBV should seek medical care from a physician with expertise in HBV management for optimal health maintenance and should be managed according to current recommendations with regular monitoring of HBV DNA levelc.

HCWs infected with HBV should be restricted from performing exposure-prone procedures until:

- the $\mathrm{HCW}$ is under the care of a physician with expertise in HBV management; and

- the HCW's HBV DNA level is below $10^{3} \mathrm{IU} / \mathrm{mL}\left(5 \times 10^{3} \mathrm{GE} / \mathrm{mL}\right)$ or equivalent and monitored regularly (every 3 to 6 months)

HCWs infected with HBV who have HBV DNA levels less than or equal to $10^{3} \mathrm{IU} / \mathrm{mL}\left(5 \times 10^{3} \mathrm{GE} / \mathrm{mL}\right)$ d or equivalent should have no restrictions on practice based on HBV status alone.

Abbreviations: anti-HBc, antibody to hepatitis $\mathrm{B}$ core antigen; anti-HBs, antibody to hepatitis $\mathrm{B}$ surface antigen; DNA, deoxyribonucleic acid; $\mathrm{HBcAg}$, hepatitis $B$ core antigen; $\mathrm{HBsAg}$, hepatitis $B$ surface antigen; HBV, hepatitis B virus; $\mathrm{HCW}$, healthcare worker

a Countries or areas with moderate to high risk of HBV are identified by the World Health Organization

b Refer to the Canadian Immunization Guide for Immunization of Persons New to Canada HBV antiviral therapy may be required to allow $\mathrm{HCW}$ infected with $\mathrm{HBV}$ to perform exposure-prone procedures

d One IU $/ \mathrm{mL}$ is considered equivalent to five copies $/ \mathrm{mL}$ (i.e. one copy $/ \mathrm{mL}$ is equivalent to $0.2 \mathrm{IU} /$ $\mathrm{mL}$ )

Because the focus is on patient safety, HCWs who perform exposure-prone procedures should be treated at this serum level regardless of recommendations in current treatment guidelines. With adherence to treatment as part of the threshold for infected HCWs to perform exposure-prone procedures, HBV DNA levels are reduced to almost undetectable in most people. As a result, several known in in various intern different assays used, or variations in results from repeated test of the same blood sample using the same assay) will likely have minima impact on decisions regarding practice restrictions 


\section{Co-infection with bloodborne viruses}

If a HCW who performs exposure-prone procedures is coinfected with any combination of HIV, HCV and/or HBV, the HCW should meet the defined criteria recommended for safe practice by HCWs infected with each virus.

\section{Double gloving}

Overall, there was insufficient evidence to recommend for or against double gloving to prevent $\mathrm{HCW}$-to-patient transmission of a BBV.

\section{Environmental scans and technical expertise}

Findings from the environmental scans and expert opinion informed the Guideline content on Expert Review Panels (ERPs) disclosure of HCWs' serologic status, and lookback investigations, with recommendations developed to fit the Canadian context. Readers interested in the full discussion on the environmental scans should refer to the Guideline (1).

\section{Expert Review Panel}

At the time of developing this guideline, not all HCWs infected with a BBV in Canada had access to an ERP. Recommendations provided for establishing ERPs address the following areas:

- Accountability, governance and membership of the ERP

- Roles and responsibilities of an ERP

- HCW referral to an ERP

- Implementation, monitoring and compliance with ERP recommendations

- Model ERP process for regulated HCWs infected with a BBV

\section{Disclosure of serologic status of healthcare workers}

Many of the current guidelines and policies that address $\mathrm{HCWs}$ and BBV infections do not contain explicit recommendations regarding disclosure obligations of HCWs to patients. Table 5 shows recommendations provided on this topic in the Guideline.

\section{Table 5: Recommendations for healthcare worker disclosure obligations and right to privacy}

\section{Recommendations}

A HCW infected with a BBV who performs exposure-prone procedures does not have an obligation to routinely disclose his or her serologic status to patients to obtain their informed consent provided that the HCW's health status and practice have been assessed by an Expert Review Panel and all the panel's recommendations are followed.

All $\mathrm{HCWs}$, including those infected with a BBV, have a right to privacy and confidentiality of personal health information.

Regulatory authorities should have policies on the management of HCWs infected with a BBV that are transparent about and detail how the right to privacy of HCWs will be upheld.

When a patient has been exposed to the blood of a HCW, the HCW must seek follow-up through their organizational process and the patient must be promptly informed of the nature of the exposure and the appropriate postexposure protocol. However, the identity and confidentiality of the HCW should be protected to the greatest extent possible.

Abbreviations: BBV bloodborne virus: $\mathrm{HCW}$, healthcare worker

\section{Lookback investigations}

Lookback investigations have been found to require significant financial and human resources to identify, notify, counsel and test all the potentially exposed patients. The Guideline provides a checklist and algorithm to help determine the requirement for a lookback investigation and considerations for conducting one

(1). These investigations may be undertaken for the following reasons:

- To notify patients of their potential exposure

- To identify infected patients and provide appropriate advice and treatment recommendations

- To prevent secondary transmission

- To reassure the public

- To maintain the public's trust and confidence in the healthcare system

- To contribute to the evidence base on risk of transmission

\section{Discussion}

This evidence-based Guideline presents a comprehensive review of relevant literature and provides national leadership on organizational policy to facilitate a consistent pan-Canadian approach to the management of HCWs infected with a BBV. It outlines steps to be taken to further reduce the minimal risk of $\mathrm{HCW}$-to-patient transmission of BBVs, striking a balance between the reasonable expectations of the public (protection from harm) and the reasonable expectations of individual HCW's (right to privacy).

All HCWs should adhere to Routine Practices, including performing hand hygiene as required and using personal protective equipment as appropriate (4). Adequate training and education on the prevention and management of occupational injuries and potential exposures are fundamental for all HCWs as part of an occupational health program. In addition, ongoing awareness of their own serologic status is essential both for optimal health maintenance for HCWs and patient safety during exposure-prone procedures.

Reports show that the average risk of transmission following exposure is highest for HBV and lowest for HIV (10). Although a $\mathrm{HCW}$ with high levels of circulating BBV may pose some risk if their blood comes in contact with a patient's open tissue, the risk approaches zero if that $\mathrm{HCW}$ is treated and eradicates $(\mathrm{HCV})$ or decreases circulating virus in their blood (HIV and HBV). In addition, with the introduction of routine hepatitis $B$ vaccination, most HCWs are protected from infection with this BBV, thus further diminishing this risk.

The Guideline applies to all HCWs, with specific recommendations for $\mathrm{HCW}$ s infected with a BBV. The recommendations are intended to assist those involved with the assessment and management of HCWs, either individually (e.g. treating physician, members of Expert Review Panels) or generally (e.g. regulatory authorities). 


\section{Conclusion}

This Guideline was developed with a rigorous methodology involving robust systematic reviews, a narrative review, environmental scans, summaries of published epidemiologic investigations, and grading of available evidence with consideration of collective expert opinion in the development of recommendations. Adhering to recommendations provided in this Guideline will result in safer practice for healthcare workers infected with a bloodborne virus. Although the Guideline reflects the latest scientific data, as new evidence is published, it may be necessary to update the applicable recommendation(s).

\section{Authors' statement}

TO - Conceptualization, project administration, methodology, research, data abstracting, writing (original draft), writing (review and editing)

$\mathrm{KD}$ - Conceptualization, project administration, research, data abstracting, writing (review and editing)

BLJ, MV, BH - Conceptualization, clinical expertise, data interpretation, intellectual content, writing (review and editing) AMJ, BC, MC, DKW, TM, JW, IB - Clinical expertise, data interpretation, intellectual content, writing (review and editing) $\mathrm{MAI}$ - Bioethics expertise, writing (review and editing)

YR - Intellectual content, review

K Dunn - Supervision, review

\section{Conflict of interest}

None.

\section{Acknowledgements}

The authors will like to thank all federal, provincial and territorial partners and key stakeholder organizations as well as subject matter experts who provided feedback on the Guideline. Refer to the Guideline for a list of organizations and individuals that were invited to provide feedback.

The authors will like to acknowledge members of Public Health Agency of Canada's National Advisory Committee on Infection Prevention and Control (NAC-IPC) for their review and feedback on this Guideline. Committee members at time of development of this Guideline were: J Embree (Chair), L Johnston (Past Chair), M Blake, G Cerkowniak, M Cividino, N Cleator, D Gregoraschuk, B Henry, J Johnstone, M Muller, H Pitfield, P Rawding, P Savard, S Smith, and J Stafford.

\section{Funding}

This work was supported by the Public Health Agency of Canada.

\section{References}

1. Public Health Agency of Canada. Guideline on the Prevention of Transmission of Bloodborne Viruses from Infected Healthcare Workers in Healthcare Settings. Ottawa (ON): PHAC; 2019. https://www.canada.ca/en/public-health/ services/infectious-diseases/nosocomial-occupationalinfections/prevention-transmission-bloodborne-viruses-h ealthcare-workers.html

2. Ogunremi T, Dunn K, Johnston L, Embree J; National Advisory Committee on Infection Prevention and Control (NAC-IPC). The National Advisory Committee on Infection Prevention and Control (NAC-IPC). Can Commun Dis Rep 2018 Nov;44(11):283-9. DOI PubMed

3. Health Canada. Division of Nosocomial and Occupational Infections. Proceedings of the Consensus Conference on Infected Health Care Workers: Risk for Transmission of Bloodborne Pathogens. Can Commun Dis Rep. 1998; Suppl 4:i-iii, 1-25; i-ii, 1-28. https://www.ncbi.nlm.nih.gov/ pubmed/11195272

4. Public Health Agency of Canada. Routine Practices and Additional Precautions for Preventing the Transmission of Infection in Healthcare Settings. Ottawa (ON): PHAC; modified 2017. https://www.canada.ca/en/public-health/ services/publications/diseases-conditions/routine-practice s-precautions-healthcare-associated-infections.html

5. Mallolas J, Arnedo M, Pumarola T, Erice A, Blanco JL, Martínez E, Gatell JM. Transmission of HIV-1 from an obstetrician to a patient during a caesarean section. AIDS 2006 Jan;20(2):285-7. DOI PubMed

6. Ross RS, Viazov S, Roggendorf M. Phylogenetic analysis indicates transmission of hepatitis $C$ virus from an infected orthopedic surgeon to a patient. J Med Virol 2002 Apr;66(4):461-7. DOI PubMed

7. Enfield KB, Sharapov U, Hall KK, Leiner J, Berg CL, Xia GL, Thompson ND, Ganova-Raeva L, Sifri CD. Transmission of hepatitis $B$ virus from an orthopedic surgeon with a high viral load. Clin Infect Dis 2013 Jan;56(2):218-24. DOI PubMed

8. Public Health Agency of Canada. Infection Prevention and Control Guidelines: Critical Appraisal Tool Kit. Ottawa (ON): PHAC; modified 2013. http://publications.gc.ca/site/ eng/470818/publication.html

9. Tanner J, Parkinson H. Double gloving to reduce surgical cross-infection [Review]. Cochrane Database Syst Rev 2002;2009(3):CD003087. DOI PubMed

10. Centers for Disease Control and Prevention. Updated U.S. Public Health Service Guidelines for the Management of Occupational Exposures to HBV, HCV, and HIV and Recommendations for Postexposure Prophylaxis. June 29, 2001. 50. (RR-11) https://www.cdc.gov/mmwr/PDF/rr/rr5011. pdf 